\title{
CCD photometric study of the short period contact binary CE Leonis
}

\author{
Yulan Yang and Qingyao Liu
}

\begin{abstract}
National Astronomical Observatories, Chinese Academy of Sciences, PR China
Yunnan Observatory, Chinese Academy of Sciences, Kunming, Yunnan Province, PR China

United Laboratory of Optical Astronomy, Chinese Academy of Sciences, PR China
\end{abstract}

Received 5 November 2001 / Accepted 4 February 2002

\begin{abstract}
Light curves and photometric solutions of the contact binary CE Leo are presented in this paper. The light curves appear to exhibit a typical O'Connell effect, with Maximum I being $0.045 \mathrm{mag}(V)$ and 0.048 mag $(B)$ brighter than Maximum II, respectively, and Maximum I shifting to phase 0.26 . The new light curve and those published by Samec et al. (1993) show the change in shape of the light curves of the system. From 1989 to 2001, the difference in the depths between the primary and secondary eclipses increased by $0.100 \mathrm{mag}(V)$ and $0.113 \mathrm{mag}(B)$. The orbital period of the system oscillates with a cycle of about 14 years and a semi-amplitude of 0.004 days. The light curves are analyzed by means of the latest version of the Wilson-Devinney code. The results show that CE Leo is a W-subtype contact binary with a ratio $q=0.501$. The asymmetry of the light curves is explained by star spot models. Variations in temperature of the spot on the primary component may be responsible for the light curve variation of the system. The cyclical period change can most probably be attributed to a magnetic activity cycle in the primary component.
\end{abstract}

Key words. stars: binaries: eclipsing - stars: magnitic fields - starspots

\section{Introduction}

The high northern latitude galactic variable (galactic latitude, $\beta=+74.31$ ), CE Leo (=S 7763), was discovered by Hoffmeister (1963) in a search for variables. This variable was originally classified RRc-type but later identified as W UMa-type by Wenzel \& Zeigler (1966). The 22 timings of minimum light and the first ephemeris were given by Meinunger \& Wenzel (1968) who gave a visual light curve of the variable. Eleven visual timings of minimum light were published in the BBSAG bulletins Nos. 9, 14, 15, 21, 37 and 92. The photometric timings of minimum light were published by Hoffmann (1983), Samec \& Bookmyer (1987), Samec \& Su (1991), Jan \& Miloslav (2000) and Nelson (2001). Samec et al. (1993) published the first complete $B V R I$ photometric light curves and the simultaneous solution of the $B, V, R, I$ light curves computed using the Wilson-Devinney synthetic light curve code. Their investigation reveals that CE Leo is a W-subtype W UMa binary consisting of two early K-type dwarfs in marginal contact with a fill-out factor less than $3 \%$. The authors also give evidence for the presence of a large $\left(45^{\circ}\right)$ superluminous area on the cooler component.

Send offprint requests to: Yulan Yang, e-mail: bily@public.km.yn.cn
Because of the large superluminous area on the cooler component and the O'Connell effect of the light curves for the system, CE Leo was included in the observing program of short period variables at the Yunnan Observatory using the 100-cm reflector telescope.

\section{Observation}

The observation of CE Leo in the $B$ and $V$ bands was carried out at 2 nights in March, 2001, with the PI1024 TKB CCD photometric system attached to the 100-cm reflecting telescope at the Yunnan Observatory in China. The effective field of view of the photometric system is $6.5 \times 6.5$ square arcminute at the Cassegrain focus and size of each pixel is 0.38 arcsec. The $B V$ color system approximates the standard Johnson $B V$ photometric system (Yang \& Li 1999). The coordinates of the variable, the comparison star and the check star used are listed in Table 1, respectively. The comparison star and the check star are the same as those used by Samec et al. (1993) and they are so close to the variable that they are in the same field of the observation together with the program star. The photometric standard stars HD 23386 , HD 23731, HD 23161 and HD 23156 were nightly observed in $B V$ bands during the observation to check the quality of the transformation coefficients of the PI1024 TKB CCD 
Table 1. The coordinates of CE Leo, comparison star and check star.

\begin{tabular}{lcc}
\hline \hline star & RA $(2000.0)$ & Dec. $(2000.0)$ \\
\hline CE Leo & $11^{\mathrm{h}} 44^{\mathrm{m}} 03^{\mathrm{s}}$ & $23^{\circ} 20^{\prime} 18^{\prime \prime}$ \\
comparison star & 114346 & 231732 \\
check star & 114341 & 232206 \\
\hline
\end{tabular}

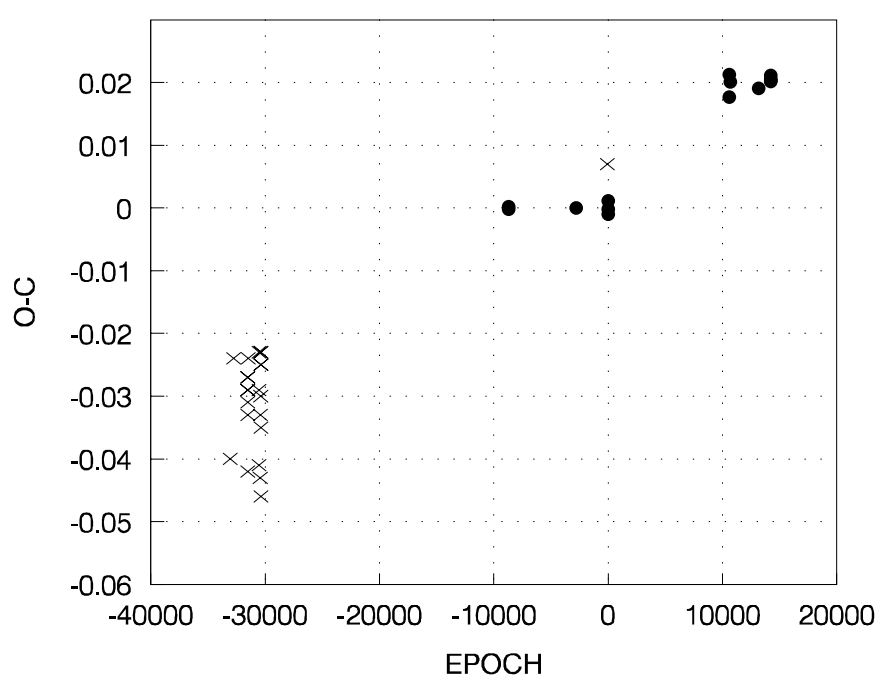

Fig. 1. Period behavior of CE Leo indicated by all available data calculated from Eq. (1). The solid circles indicate photoelectric data and the crosses show the visual or photographic ones.

photometric system of the $100-\mathrm{cm}$ reflecting telescope to those of the Johnson standard $U B V$ system.

The integration time for each image is $100 \mathrm{~s}$. A total of 156 images in $V$ band and 157 images in $B$ band was obtained for 2 nights in March, 2001. The aperture photometry package of IRAF was used to reduce the images. The reduced results show that the difference between the magnitude of the check star and that of the comparison star is constant within a probable error of \pm 0.005 mag for $V$ color and of \pm 0.006 mag for $B$ color. Differential extinction corrections were found to be negligible since the comparison star is very close to the variable and were not applied to the observations.

From the observations during two primary and two secondary eclipses, four epochs of minimum light were derived by using parabola fitting. The new timings of the minimum light together with those published by other authors are listed in Table 2 , in which the $(\mathrm{O}-\mathrm{C})_{1}$ values are calculated by means of the light element formula given in the Samec et al. (1993).

Min.I = HJD 2447679.6689+0 $\mathrm{d} 30342785 E$.

The $(\mathrm{O}-\mathrm{C})_{1}$ values computed with the Eq. (1) are plotted in Fig. 1. This diagram shows that the orbital period of $\mathrm{CE}$ Leo seems to be long-term stable. We fit the photoelectric timings weighted by 1.0 and all others weighted by 0.1 by
Table 2. The timings of minimum light of CE Leo.

\begin{tabular}{|c|c|c|c|c|}
\hline HJD 2400000+ & Min. & $(\mathrm{O}-\mathrm{C}) 1$ & $(\mathrm{O}-\mathrm{C}) 2$ & reference \\
\hline 37651.642 & $\bar{I}$ & -0.040 & -0.007 & Meinunger \& Wenzel (1968) \\
\hline 37737.528 & I & -0.024 & 0.009 & Meinunger \& Wenzel (1968) \\
\hline 38111.500 & II & -0.027 & 0.005 & Meinunger \& Wenzel (1968) \\
\hline 38112.410 & II & -0.027 & 0.004 & Meinunger \& Wenzel (1968) \\
\hline 38112.560 & I & -0.029 & 0.003 & Meinunger \& Wenzel (1968) \\
\hline 38113.470 & I & -0.029 & 0.002 & Meinunger \& Wenzel (1968) \\
\hline 38113.620 & II & -0.031 & 0.001 & Meinunger \& Wenzel (1968) \\
\hline 38116.340 & II & -0.042 & -0.010 & Meinunger \& Wenzel (1968) \\
\hline 38113.500 & I & -0.033 & -0.002 & Meinunger \& Wenzel (1968) \\
\hline 38134.412 & I & -0.024 & 0.009 & Meinunger \& Wenzel (1968) \\
\hline 38412.650 & I & -0.029 & 0.001 & Meinunger \& Wenzel (1968) \\
\hline 38414.610 & II & -0.041 & -0.011 & Meinunger \& Wenzel (1968) \\
\hline 38415.690 & I & -0.023 & 0.007 & Meinunger \& Wenzel (1968) \\
\hline 38446.620 & I & -0.043 & -0.013 & Meinunger \& Wenzel (1968) \\
\hline 38457.402 & II & -0.033 & -0.002 & Meinunger \& Wenzel (1968) \\
\hline 38458.315 & II & -0.030 & 0.000 & Meinunger \& Wenzel (1968) \\
\hline 38463.480 & II & -0.023 & 0.007 & Meinunger \& Wenzel (1968) \\
\hline 38463.620 & I & -0.035 & -0.005 & Meinunger \& Wenzel (1968) \\
\hline 38464.390 & II & -0.023 & 0.007 & Meinunger \& Wenzel (1968) \\
\hline 38464.540 & I & -0.025 & 0.005 & Meinunger \& Wenzel (1968) \\
\hline 38465.450 & I & -0.025 & 0.005 & Meinunger \& Wenzel (1968) \\
\hline 38466.340 & I & -0.046 & -0.046 & Meinunger \& Wenzel (1968) \\
\hline 45044.5495 & II & -0.0002 & 0.0051 & Meinunger \& Wenzel (1968) \\
\hline 45047.4325 & I & 0.0002 & 0.0055 & Hoffmann (1983) \\
\hline 46829.0089 & II & 0.0000 & -0.0015 & Samec \& Bookmyer (1987) \\
\hline 47655.402 & I & 0.007 & 0.003 & BBSAG 92 (1989) \\
\hline 47679.6687 & I & -0.0002 & -0.0049 & Samec et al. (1993) \\
\hline 47680.7320 & II & 0.0011 & -0.0036 & Samec et al. (1993) \\
\hline 47683.7642 & II & -0.0010 & -0.0057 & Samec et al. (1993) \\
\hline 50888.2880 & II & 0.0213 & 0.0044 & Safar \& Zejda (2000) \\
\hline 50895.4150 & I & 0.0177 & 0.0009 & Safar \& Zejda (2000) \\
\hline 50921.3604 & II & 0.0201 & 0.0031 & Safar \& Zejda (2000) \\
\hline 51672.79847 & I & 0.0191 & -0.0007 & Nelson (2001) \\
\hline 51995.1917 & II & 0.0202 & -0.0009 & this paper \\
\hline 51995.3435 & I & 0.0203 & -0.0008 & this paper \\
\hline 51996.1024 & II & 0.0206 & -0.0004 & this paper \\
\hline 51996.2546 & I & 0.0211 & 0.0001 & this paper \\
\hline
\end{tabular}

a linear least squares and obtain a improved ephemeris as follows:

Min.I = HJD 2447679.6736(5) + 0.30342900(5)E,

which is used to compute the phases of our observations and the $(\mathrm{O}-\mathrm{C})_{2}$ values in Table 2 . From the $(\mathrm{O}-\mathrm{C})_{2}$ values, one can see that short-term changes in the orbital period of CE Leo seem to occur. The $(\mathrm{O}-\mathrm{C})_{2}$ values of the photoelectric epochs versus their epochs are shown in Fig. 2 by solid circles. The solid curve plotted in Fig. 2 indicates a result of fitting the observations by a least squares method. From Fig. 2, one can see that a cyclical change in the orbital period of CE Leo seems to occur. The orbital period of CE Leo oscillates with a cycle of about 14 years and an semi-amplitude of 0.004 days.

A total of 156 yellow and 157 blue observations has been obtained and listed in Tables 4 and 5 with their Heliocentric Julian Days, phases and magnitude differences between the variable and the comparison star. The light curve of the system is shown as the solid circle points in Fig. 2. The light curves appear to exhibit a typical O'Connell effect, with Maximum I being $0.045 \mathrm{mag}(V)$ 


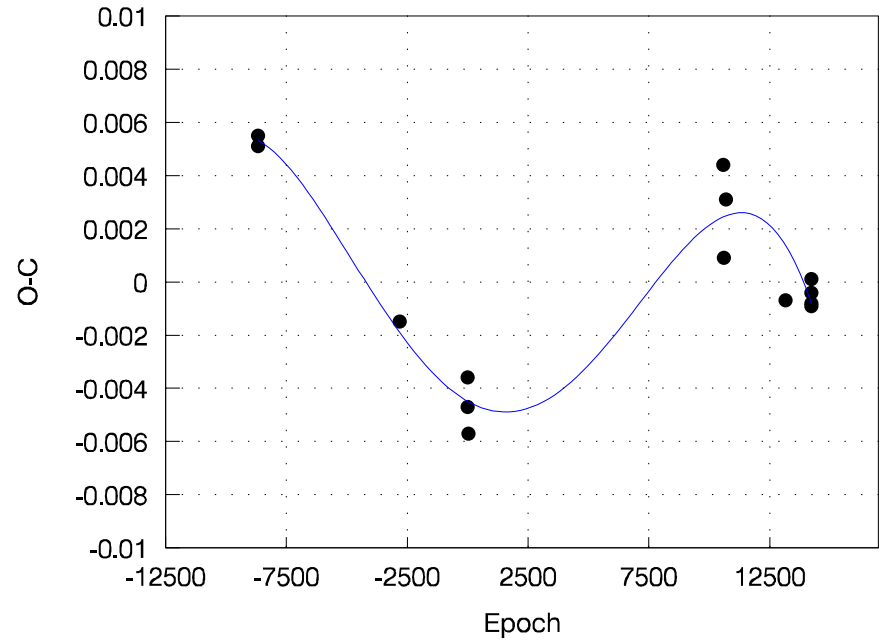

Fig. 2. Periodic change in the orbital period of CE Leo.

Table 3. The parameters of the light curve properties of CE Leo.

\begin{tabular}{lccc}
\hline \hline obs. date & 1989 & 2001 & diff. \\
\hline Max.I-Min.I in $V$ & -0.841 & -0.893 & 0.052 \\
Max.I-Min.I in $B$ & -0.900 & -0.955 & 0.055 \\
Max.I-Min.II in $V$ & -0.737 & -0.689 & -0.048 \\
Max.I-Min.II in $B$ & -0.785 & -0.727 & -0.058 \\
Max.II-Min.I in $V$ & -0.778 & -0.848 & 0.070 \\
Max.II-Min.I in $B$ & -0.846 & -0.907 & 0.061 \\
Max.II-Min.II in $V$ & -0.674 & -0.644 & -0.030 \\
Max.II-Min.II in $B$ & -0.731 & -0.679 & -0.052 \\
Max.I-Max.II in $V$ & -0.063 & -0.045 & -0.018 \\
Max.I-Max.II in $B$ & -0.054 & -0.048 & -0.006 \\
Min.I-Min.II in $V$ & 0.104 & 0.204 & -0.100 \\
Min.I-Min.II in $B$ & 0.115 & 0.228 & -0.113 \\
\hline
\end{tabular}

and $0.048 \mathrm{mag}(B)$ brighter than Maximum II, respectively, and Maximum I shifting to phase 0.26. In order to investigate variations in the light curves of the system, a graphical depiction of the variable shape of the light curves in $V$ band is shown in Fig. 3, which gives the observations carried out by Samec et al. (1993) (X's) in 1989 and the present ones (solid circles). As shown in Fig. 3, the variation of the light curve of the system is very obvious. Some parameters of the light curves for CE Leo are listed in Table 3, from which one can see that the difference of the eclipsing depth between the primary eclipse and the secondary one is considerably variable. The depth of the primary eclipse (Max.I-Min.I or Max.II-Min.I) increased on average about $0.061 \mathrm{mag}(V)$ and $0.058 \mathrm{mag}(B)$, respectively, but the depth of the secondary eclipse (Max.I-Min.II or Max.II-Min.II) decreased by about $0.039 \mathrm{mag}(V)$ and $0.055 \mathrm{mag}(B)$, on average. Therefore, the difference of the depth between the primary eclipse and the secondary one increased by about $0.100 \mathrm{mag}(V)$ and $0.113 \mathrm{mag}(B)$, respectively.

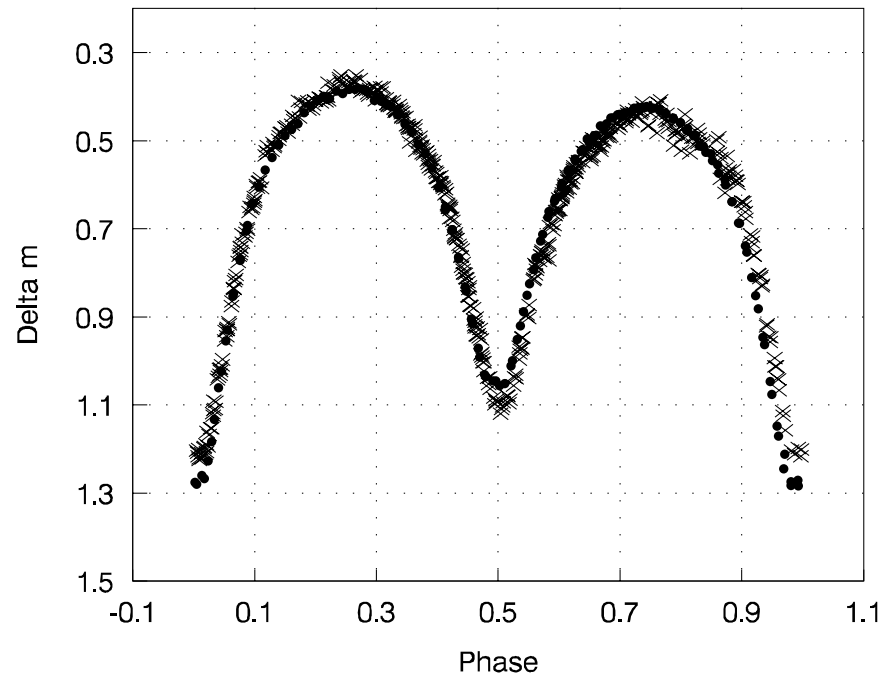

Fig. 3. The variation of the light curves of CE Leo. The crosses indicate the observations obtained by Samec et al. in 1989 and the solid circles show the present data.

The usual standardization procedure of the observations of the CE Leo outside eclipse to the Johnson's standard $U B V$ system (Johnson 1963; Landolt 1973, 1983) is made to obtain the $B-V$ color. The transformation coefficients are determined by the observations of the standard stars (Yang \& Li 1999). In order to check the quality of the transformation coefficients, the standardization procedure of the observations of the observed photometric standard stars HD 23386, HD 23731, HD 23161 and HD 23156 was made. The results show that the standardization procedure is applicable to the Johnson's standard $U B V$ system with the transformation coefficients of the PI1024 TKB CCD photometric system of the $100-\mathrm{cm}$ reflecting telescope at the Yunnan Observatory. No significant systematic difference appeared between the two systems. The maximum deviation of the standardization procedure is $\pm 0.008 \mathrm{mag}$ for $V$ and $\pm 0.010 \mathrm{mag}$ for $B$. The determined $B-V$ color of CE Leo is +0.865 at the phase of 0.25 and +0.873 at the phase of 0.75 , which is very close to 0.871 at the phase of 0.25 and 0.882 at the phase of 0.75 obtained by Samec et al. (1993).

\section{Photometric solutions}

Photometric solutions are obtained by means of the Wilson-Devinney (Wilson 1994) Code which includes a new reflection treatment, the option of using non-linear limb-darkening laws and the ability to adjust spot parameters. All the observations are used to compute the solutions. The convergence of the minimization procedure is obtained by means of the method of multiple subsets (Wilson \& Biermann 1976).

The adopted parameters in the solutions are described as follows: a temperature of $5150 \mathrm{~K}$ for Star 1 (the star eclipsed at Min.I), which corresponds to the $B-V$ color of 0.87 (Samec et al. 1993), the values of the limb darkening coefficients $\left(x_{1}=x_{2}=0.710\right.$ for $V$ band and 0.860 for 
Table 4. The CCD observations in $V$ band for CE Leo.

\begin{tabular}{|c|c|c|c|c|c|c|c|c|c|}
\hline JD.(HEL) & $\Delta m$ & JD.(HEL) & $\Delta m$ & JD.(HEL) & $\Delta m$ & JD.(HEL) & $\Delta m$ & JD.(HEL) & $\Delta m$ \\
\hline $2451990+$ & & $2451990+$ & & $2451990+$ & & $2451990+$ & & $2451990+$ & \\
\hline 5.1183 & .382 & 5.2380 & .504 & 5.3532 & 1.183 & 6.1601 & .448 & 6.2649 & 1.133 \\
\hline .1265 & .385 & .2414 & .487 & .3567 & 1.061 & .1632 & .440 & .2681 & 1.023 \\
\hline .1322 & .400 & .2449 & .472 & .3603 & .955 & .1664 & .438 & .2713 & .930 \\
\hline .1357 & .413 & .2486 & .449 & .3638 & .853 & .1706 & .426 & .2744 & .847 \\
\hline .1391 & .422 & .2521 & .442 & .3674 & .772 & .1738 & .425 & .2778 & .768 \\
\hline .1425 & .442 & .2555 & .437 & .3710 & .693 & .1769 & .423 & .2809 & .704 \\
\hline .1461 & .463 & .2590 & .433 & 6.0735 & .607 & .1801 & .426 & .2841 & .642 \\
\hline .1494 & .480 & .2624 & .425 & .0767 & .650 & .1833 & .432 & .2872 & .606 \\
\hline .1528 & .508 & .2658 & .425 & .0799 & .702 & .1864 & .439 & .2903 & .566 \\
\hline .1562 & .530 & .2692 & .426 & .0831 & .765 & .1904 & .451 & .2936 & .538 \\
\hline .1595 & .562 & .2728 & .429 & .0864 & .832 & .1936 & .459 & .2968 & .510 \\
\hline .1630 & .607 & .2762 & .438 & .0896 & .905 & .1968 & .477 & .3001 & .489 \\
\hline .1663 & .657 & .2797 & .448 & .0928 & .971 & .2001 & .488 & .3033 & .475 \\
\hline .1697 & .702 & .2834 & .459 & .0959 & 1.031 & .2032 & .512 & .3064 & .461 \\
\hline .1731 & .768 & .2870 & .472 & .1018 & 1.045 & .2064 & .526 & .3096 & .437 \\
\hline .1766 & .842 & .2910 & .488 & .1060 & 1.051 & .2096 & .545 & .3129 & .421 \\
\hline .1799 & .916 & .2949 & .515 & .1092 & 1.011 & .2128 & .574 & .3160 & .409 \\
\hline .1833 & .991 & .2985 & .530 & .1124 & .951 & .2162 & .601 & .3193 & .399 \\
\hline .1866 & 1.036 & .3021 & .555 & .1155 & .889 & .2194 & .638 & .3224 & .405 \\
\hline .1900 & 1.046 & .3056 & .588 & .1186 & .825 & .2226 & .687 & .3256 & .387 \\
\hline .1932 & 1.056 & .3093 & .638 & .1218 & .766 & .2260 & .739 & .3287 & .393 \\
\hline .1996 & 1.000 & .3130 & .688 & .1250 & .713 & .2292 & .811 & .3318 & .384 \\
\hline .2037 & .920 & .3164 & .753 & .1282 & .661 & .2324 & .881 & .3350 & .384 \\
\hline .2071 & .851 & .3208 & .852 & .1313 & .631 & .2355 & .963 & .3383 & .383 \\
\hline .2104 & .793 & .3246 & .946 & .1345 & .597 & .2392 & 1.076 & .3414 & .389 \\
\hline .2138 & .728 & .3281 & 1.047 & .1379 & .566 & .2426 & 1.171 & .3446 & .408 \\
\hline .2173 & .674 & .3315 & 1.148 & .1411 & .542 & .2458 & 1.212 & .3479 & .411 \\
\hline .2207 & .637 & .3349 & 1.245 & .1442 & .524 & .2489 & 1.283 & 6.3510 & .419 \\
\hline .2241 & .606 & .3386 & 1.275 & .1474 & .496 & .2521 & 1.271 & & \\
\hline .2274 & .568 & .3421 & 1.284 & .1506 & .489 & .2552 & 1.276 & & \\
\hline .2311 & .545 & .3457 & 1.280 & .1538 & .466 & .2585 & 1.260 & & \\
\hline 5.2346 & .521 & 5.3495 & 1.267 & 6.1569 & .462 & 6.2617 & 1.227 & & \\
\hline
\end{tabular}

$B$ band) (Claret \& Gimenez 1990), the values of the gravity darkening coefficients $\left(g_{1}=g_{2}=0.320\right)$ (Lucy 1967) and the values of the albedo $\left(A_{1}=A_{2}=0.500\right)$ (Rucinski 1969), which corresponds to the $B-V$ color of 0.87 for $\mathrm{CE}$ Leo. The adjustable parameters are the orbital inclination, $\iota$, the mean temperature of Star $2, T_{2}$, the potential of the components, $\Omega_{1}$ and $\Omega_{2}$, and the monochromatic luminosity of Star $1, L_{1}$ (the Planck function is used to compute the luminosity).

Solutions are worked out for a series of fixed values of the mass ratio $q=m_{2} / m_{1}(0.20,0.30,0.40,0.50,0.60$, $0.70,0.80,0.90,0.10,1.40,1.60,2.00,2.40,2.60,3.00,3.40$, 4.00). Assuming that initially it was a detached system, the differential corrections started from Mode 2, but the converged solutions were always obtained from the contact Mode 3 . The resulting sum $\Sigma$ of the square deviations of the converged solutions for each value of $q$ indicates that the fitting is best for $q=2.00$. At this point, the set of the adjustable parameters is expanded to include $q$. The mass ratio converges to a value of $q=1.99514$ in the final solution. The filling factor, $f=0.034$, has here the expression $\left(\Omega_{\text {in }}-\Omega\right) /\left(\Omega_{\text {in }}-\Omega_{\text {out }}\right)$ and varies from 0 to unity from the inner to the outer critical surface. This solution indicates that CE Leo is a W-subtype W UMa binary with a shallow contact degree. The photometric parameters are listed in the second column (unspotted) of Table 6 . The light curve computed using these parameters in the second column of the Table 6 is shown by the solid lines in Fig. 4.

While the overall fit of the computed light curves is satisfactory, Fig. 4 shows obvious distortions in the observed light curves that seem to be due to the surface inhomogeneities of the components. Unequal quadrature light level, namely, the O'Connell effect, is known in many 
Table 5. The CCD observations in $B$ band for CE Leo.

\begin{tabular}{|c|c|c|c|c|c|c|c|c|c|}
\hline JD.(HEL) & $\Delta m$ & JD.(HEL) & $\Delta m$ & JD.(HEL) & $\Delta m$ & JD.(HEL) & $\Delta m$ & JD.(HEL) & $\Delta m$ \\
\hline $2451990+$ & & $2451990+$ & & $2451990+$ & & $2451990+$ & & $2451990+$ & \\
\hline 5.1233 & .314 & 5.2363 & .446 & 5.3477 & 1.243 & 6.1554 & .392 & 6.2601 & 1.246 \\
\hline .1286 & .310 & .2397 & .421 & .3513 & 1.206 & .1585 & .383 & .2633 & 1.158 \\
\hline .1304 & .318 & .2432 & .407 & .3549 & 1.101 & .1617 & .373 & .2665 & 1.059 \\
\hline .1341 & .340 & .2467 & .387 & .3584 & .960 & .1648 & .357 & .2697 & .932 \\
\hline .1374 & .340 & .2504 & .363 & .3621 & .849 & .1691 & .354 & .2729 & .842 \\
\hline .1408 & .371 & .2538 & .360 & .3656 & .769 & .1722 & .349 & .2761 & .739 \\
\hline .1444 & .377 & .2573 & .346 & .3692 & .669 & .1754 & .343 & .2794 & .675 \\
\hline .1477 & .404 & .2607 & .352 & .0712 & .524 & .1785 & .343 & .2825 & .608 \\
\hline .1511 & .421 & .2642 & .350 & .0751 & .573 & .1817 & .359 & .2857 & .563 \\
\hline .1545 & .449 & .2642 & .350 & .0784 & .624 & .1849 & .351 & .2888 & .521 \\
\hline .1579 & .482 & .2675 & .336 & .0815 & .678 & .1880 & .366 & .2920 & .485 \\
\hline .1612 & .516 & .2710 & .343 & .0848 & .754 & .1921 & .384 & .2951 & .458 \\
\hline .1646 & .556 & .2745 & .348 & .0880 & .812 & .1952 & .396 & .2986 & .429 \\
\hline .1681 & .606 & .2780 & .359 & .0912 & .894 & .1984 & .415 & .3017 & .416 \\
\hline .1714 & .680 & .2816 & .370 & .0944 & .971 & .2017 & .425 & .3049 & .387 \\
\hline .1749 & .742 & .2851 & .397 & .1002 & 1.017 & .2049 & .449 & .3080 & .372 \\
\hline .1783 & .822 & .2887 & .423 & .1057 & 1.011 & .2081 & .462 & .3112 & .349 \\
\hline .1816 & .903 & .2932 & .428 & .1076 & .984 & .2113 & .487 & .3145 & .335 \\
\hline .1850 & .972 & .2968 & .453 & .1108 & .939 & .2145 & .514 & .3176 & .335 \\
\hline .1883 & 1.003 & .3002 & .470 & .1140 & .871 & .2178 & .546 & .3208 & .309 \\
\hline .1916 & 1.011 & .3038 & .515 & .1171 & .812 & .2210 & .590 & .3241 & .314 \\
\hline .1989 & .985 & .3076 & .553 & .1202 & .752 & .2242 & .639 & .3272 & .303 \\
\hline .2020 & .905 & .3112 & .597 & .1234 & .692 & .2276 & .717 & .3303 & .295 \\
\hline .2054 & .825 & .3147 & .669 & .1266 & .642 & .2308 & .787 & .3334 & .294 \\
\hline .2087 & .766 & .3183 & .726 & .1298 & .598 & .2340 & .868 & .3366 & .313 \\
\hline .2122 & .707 & .3227 & .857 & .1329 & .559 & .2372 & .966 & .3399 & .314 \\
\hline .2155 & .646 & .3264 & .960 & .1363 & .518 & .2410 & 1.085 & .3431 & .339 \\
\hline .2190 & .593 & .3298 & 1.068 & .1395 & .489 & .2442 & 1.197 & .3462 & .342 \\
\hline .2225 & .555 & .3332 & 1.180 & .1427 & .469 & .2474 & 1.255 & 6.3494 & .348 \\
\hline .2257 & .525 & .3369 & 1.250 & .1459 & .443 & .2505 & 1.257 & & \\
\hline .2294 & .492 & .3404 & 1.261 & .1490 & .438 & .2536 & 1.247 & & \\
\hline 5.2329 & .462 & 5.3440 & 1.265 & 6.1522 & .417 & 6.2569 & 1.254 & & \\
\hline
\end{tabular}

eclipsing binaries and several suggestions have been made to explain this effect by various authors. For CE Leo, the observed distortion, with Maximum II being fainter than Maximum I, may result from a cool or a hot region on either component. It is assumed that the spot is on star 1 (the star eclipsed at Min.I) or star 2 and several groups of dark spots or hot spots are tested. Finally, four converged solutions are found with a cool spot on star 1 or star 2 and with a hot spot on star 1 or star 2 , respectively. The solutions with the spots are also listed in Table 6 , where the solution labeled Dark 1 has a cool spot on the primary (more massive) star, labeled Dark 2 with a cool spot on the secondary star, the one labeled Hot 1 has a hot spot on the primary star and the one labeled Hot 2 has a hot spot on the secondary star. Also listed in Table 6 are spot labels and parameters (co-latitude, $\theta$, longitude, $\phi$, angular radius $\gamma$, all in degrees, and the temperature factor $T_{\mathrm{s}} / T_{*}$, with $T_{*}$ being the local effective temperature of the surrounding photosphere). As shown by Maceroni \& van't Veer (1993), spot determination by photometry alone is unreliable because of the uniqueness of photometric solutions for spotted W UMa-type binaries. The spot parameters listed in Table 6 are temporary, but the solution labelled Dark 1, i.e. the one with a cool spot on the primary (=more massive) star, turns out to be of obviously better quality than the others. The corresponding computed light curves are shown by the solid lines in Fig. 5. From Fig. 5 alone we would conclude that the O'Connell effect for CE Leo is due to a cool area appearing on the surface of the primary component. 
Table 6. The photometric solutions of CE Leo.

\begin{tabular}{lccccc}
\hline \hline parameters & unspotted & Dark 1 & Dark 2 & Hot 1 & Hot 2 \\
\hline$q=m_{2} / m_{1}$ & $0.5012(14)$ & $0.5014(12)$ & $0.5043(14)$ & $0.4946(15)$ & $0.4932(16)$ \\
$i$ & $86.44(24)$ & $87.01(21)$ & $87.83(29)$ & $85.08(32)$ & $85.88(28)$ \\
$T_{1}(K)$ & $4754(5)$ & $4811(2)$ & $4765(3)$ & $4725(2)$ & $4762(3)$ \\
$T_{2}(K)$ & 5150 & 5150 & 5150 & 5150 & 5150 \\
$\Omega$ & $2.8680(37)$ & $2.8656(13)$ & $2.8683(16)$ & $2.8556(15)$ & $2.8357(18)$ \\
$f$ & 0.034 & 0.043 & 0.052 & 0.033 & 0.026 \\
$L_{1 V} /\left(L_{1 V}+L_{2 V}\right)$ & $0.5493(12)$ & $0.5652(5)$ & $0.5491(7)$ & $0.5439(5)$ & $0.5599(6)$ \\
$L_{1 B} /\left(L_{1 B}+L_{2 B}\right)$ & $0.5262(14)$ & $0.5459(6)$ & $0.5239(9)$ & $0.5159(5)$ & $0.5345(6)$ \\
$x_{1 V}=x_{2 V}$ & 0.710 & 0.710 & 0.710 & 0.710 & 0.710 \\
$x_{1 B}=x_{2 B}$ & 0.860 & 0.860 & 0.860 & 0.860 & 0.860 \\
$A_{1}=A_{2}$ & 0.500 & 0.500 & 0.500 & 0.500 & 0.500 \\
$g_{1}=g_{2}$ & 0.320 & 0.320 & 0.320 & 0.320 & 0.320 \\
$r_{1}$ (pole) & $0.4218(6)$ & $0.4237(2)$ & $0.4255(2)$ & $0.4227(5)$ & $0.4233(6)$ \\
$r_{1}$ (side) & $0.4497(8)$ & $0.4522(3)$ & $0.4546(3)$ & $0.4508(7)$ & $0.4513(8)$ \\
$r_{1}$ (back) & $0.4809(10)$ & $0.4842(4)$ & $0.4878(5)$ & $0.4818(9)$ & $0.4815(11)$ \\
$r_{2}$ (pole) & $0.3080(7)$ & $0.3100(2)$ & $0.3129(3)$ & $0.3067(4)$ & $0.3035(4)$ \\
$r_{2}$ (side) & $0.3227(7)$ & $0.3252(3)$ & $0.3285(3)$ & $0.3213(5)$ & $0.3177(5)$ \\
$r_{2}$ (back) & $0.3606(12)$ & $0.3646(4)$ & $0.3699(6)$ & $0.3591(8)$ & $0.3545(9)$ \\
$\Sigma$ & 0.013871 & 0.003077 & 0.004959 & 0.007586 & 0.007826 \\
$\phi$ & & $89.3(6)$ & $89.1(7)$ & $87.7(8)$ & $97.2(8)$ \\
$\theta$ & & $233.3(9)$ & $75.3(6)$ & $117.0(9)$ & $286.2(7)$ \\
$\gamma$ & & $18.7(4)$ & $20.5(3)$ & $18.9(3)$ & $21.5(3)$ \\
$T_{\mathrm{s}} / T_{*}$ & $0.80(2)$ & $0.80(3)$ & $1.10(2)$ & $1.10(3)$ \\
\hline & & & & &
\end{tabular}

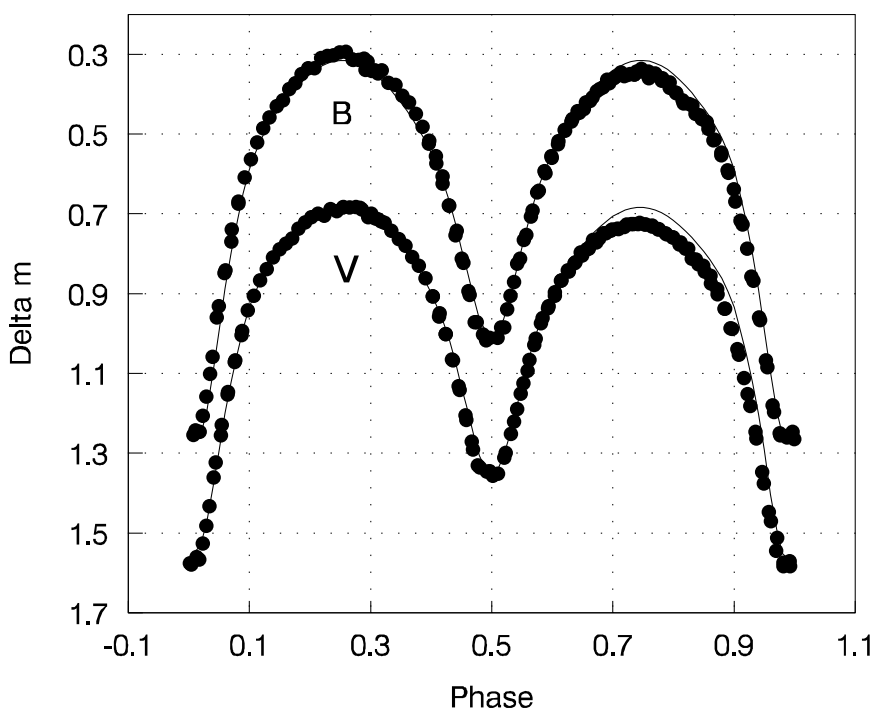

Fig. 4. The light curves of CE Leo. Solid circles: observational data; lines: model (see text for details).

\section{Discussion}

CE Leo is a very short-period W UMa-type contact binary consisting of two early spectral K-type stars. The present CCD photometric study suggests that the periodical change in the orbital period of the system with a

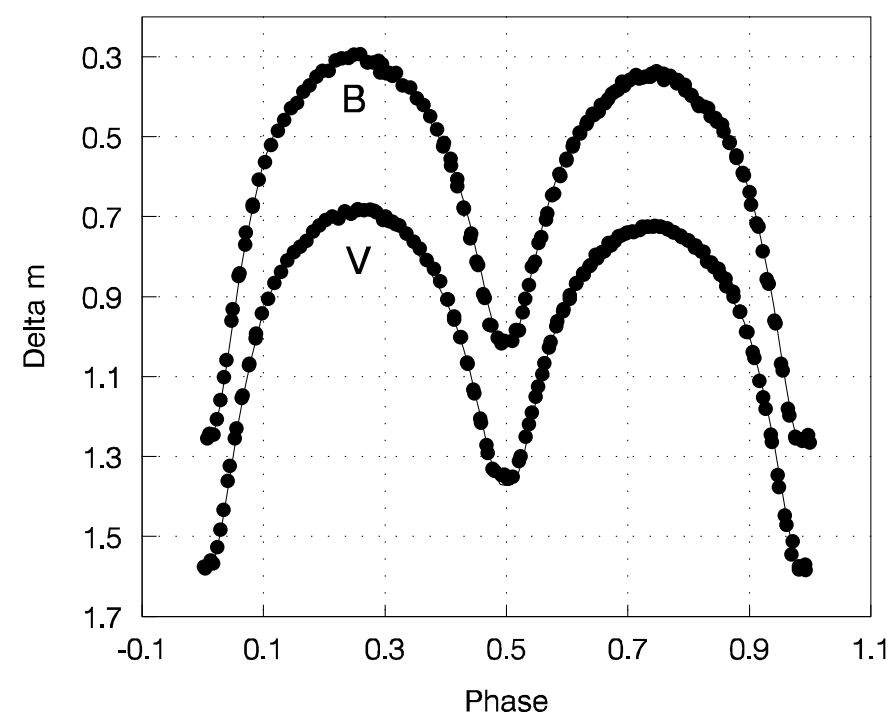

Fig. 5. As Fig. 4, but the lines derived from the model with a dark spot on the primary component.

short-term cycle of 14 years and the variable difference of the eclipsing depth between the primary eclipse and the secondary one may be due to cyclical spot-activity on the primary component of the system.

Maceroni \& van't Veer (1996) gave the absolute dimensions of CE Leo. The mass of $0.94 m_{\odot}$ and the radius 
of $0.94 R_{\odot}$ for the primary component suggest that the primary of the system is a late-type main-sequence star with rapid rotation. Therefore, violent magnetic activity on the component can be reasonably expected. The solutions obtained by Samec et al. (1993) from their complete $V B R I$ light curves show the possible presence of a large hot spot region on the primary component of the system. The present solution with lowest residual, however, suggests the presence of a cool spot region at almost the same position as the hot spot region. This transition from the hot spot to the cool spot on the primary component may be the origin of the variation of the light curves of the system. CE Leo is a W-subtype W UMa contact binary, i.e. the primary eclipse is the occultation but the secondary eclipse is the transit. Both the increase of the depth of the primary eclipse and the decrease of the depth of the secondary eclipse from 1989 to 2001 suggest decrease of the surface temperature on the primary component. The temperature difference of the two components, $\delta T=260$, in Samec et al.'s solution is lower than $\delta T=339$ in the present solution. The average temperature of the primary component decreased about $80 \mathrm{~K}$ degree due to the change in the spot temperature.

Alternating period changes are observed in many W UMa-type binaries (Kreiner 1977) as well as in most binaries of other types containing at least one convective component (Tout \& Hall 1991; Hall 1990). Both the effect of light-time due to the third body and the result from magnetic cycles in a star with an active dynamo (Hall 1991) can be responsible for the alternating period changes of the binaries. For $\mathrm{CE}$ Leo, since the $\mathrm{O}-\mathrm{C}$ curve (see Fig. 2) of the cyclical period change is not a normal sine one, its alternating period change probably can not be attributed to the light-time effect due to a hypothetical third body, but can most probably be attributed to a magnetic activity cycle in the primary component. If so, the period of the magnetic activity cycle may be about 14 years.

Since the nature of the light curve variation and the orbital period change of CE Leo is very important to our understanding of magnetic activity in W UMa-type binaries, we invite photometric observations of this system over the next few decades. Also, spectroscopic observations will be necessary.

Acknowledgements. The authors would like to thank Mr. J. Li for his assistance in the observations. The authors would also like to express their gratitude for the support from the Chinese National Science Foundation Committee and the Chinese Academy of Sciences.

\section{References}

Claret, A., \& Gimenez, A. 1990, A\&A, 230, 412

Hall, D. S. 1990, in Active Close Binaries, ed. C. Ibanoglu (Kluwer, Dordrecht), 95

Hall, D. S. 1991, ApJ, 380, L85

Hoffmann, M. 1983, IBVS No. 2344

Hoffmeister, C. 1963, AN, 287, 169

Johnson, H. L. 1963, In Basic Astronomical Data, ed. K. Aa. Strand (University of Chicago, Chicago), 204

Kreiner, J. M. 1977, IAU Colloq., 42, 373

Landolt, A. U. 1973, AJ, 78, 959

Landolt, A. U. 1983, AJ, 88, 439

Lucy, L. B. 1967, Zert. Astrophys., 65, 89

Maceroni, C., \& van't Veer, F. 1996, A\&A, 311, 523

Maceroni, C., \& van't Veer, F. 1993, A\&A, 277, 515

Meinunger, L., \& Wenzel, W. 1968, VSS 7(4), 430

Nelson, R. H. 2001, IBVS, No. 5040

Rucinski, S. M. 1969, A\&A, 19, 245

Safar, J., \& Zejda, M. 2000, IBVS, No. 4888

Samec, R. G., \& Bookmyer, B. B. 1987, IBVS, No. 3053

Samec, R. G., \& Su, W. 1991, IBVS, No. 3650

Samec, R. G., Su, W., Terrell, D., \& Hube, D. P. 1993, AJ, 106,318

Tout, C. A., \& Hall, D. S. 1991, MNRAS, 253, 9

Wenzel, W., \& Zeigler, E. 1966, MVS 4(2), 20

Wilson, R. E., \& Biermann 1976, A\&A, 48, 349

Wilson, R. E. 1994, PASP, 106, 921

Yang, Y., \& Li, L. 1999, Publication of Yunnan Observatory, 1,32 